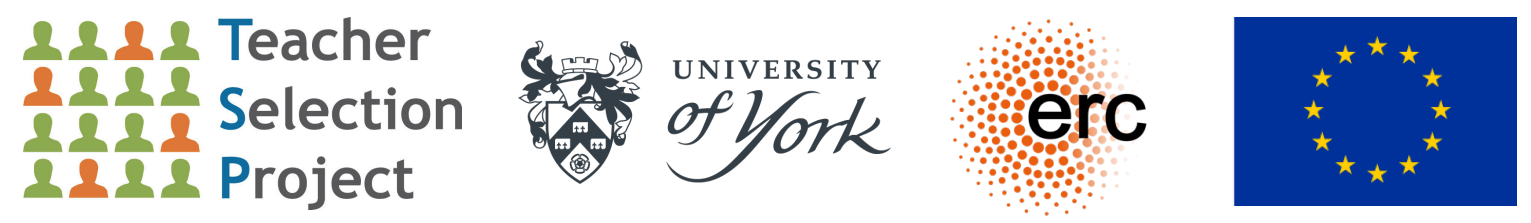

Teacher Selection Project Working Paper

04.10 .18

Development of an Online Construct-Informed Situational Judgment Test for Screening Applicants for Initial Teacher Education

Robert M. Klassen and Lisa E. Kim University of York 


\title{
Development of an Online Construct-Informed Situational Judgment Test for Screening Applicants for Initial Teacher Education
}

\author{
Robert M. Klassen and Lisa E. Kim \\ University of York
}

\begin{abstract}
The purpose of this article is to report three phases of development and administration of an online situational judgment test (SJT) designed to screen candidates for selection into an initial teacher education (ITE) program. In Phase 1 the development of the content of the test is described. Phase 2 reports the online administration of a prototype SJT to 3341 applicants as part of the process designed to select candidates for an intensive day-long assessment center. Phase 3 reports the administration of a revised version of the SJT to 587 participants. Results showe that the revised SJT was internally reliable and significantly related to other screening methods and to assessment center outcomes. High scorers on the SJT scored significantly higher at the assessment center. There were no significant SES differences on the SJT, but females scored significantly higher than males. The factor structure of the test was unidimensional and did not cleanly reflect the six target attributes which underpinned the development of test content. We suggest that the teacher selection SJT could be a reliable, valid, and efficient screening tool for entrance into ITE.
\end{abstract}

\section{Introduction}

One of the first steps in the development of an effective teacher workforce is to identify candidates who first, are likely to succeed in an initial teacher education (ITE) program, and second, are likely to experience success as practicing teachers. Evidence for individual differences in the developmental trajectory of teachers is persuasive (Atteberry, Loeb, \& Wyckoff, 2015; Chetty, Friedman, \& Rockoff, 2014; Hanushek \& Rivkin, 2012; Xu, Özek, \& Hansen, 2015), with both cognitive attributes (e.g., academic ability, subject knowledge, pedagogical knowledge) and non-cognitive attributes (e.g., interpersonal skills, personality, and motivation) contributing to these differences (Klassen \& Tze, 2014; Rockoff, Jacob, Kane, \& Staiger, 2011). The contribution of teachers' non-cognitive attributes can be traced to the very beginning stages of training and professional practice (Bastian, McCord, Marks, \& Carpenter, 2017; Watt, Richardson, \& Wilkins, 2014), but identifying and assessing these attributes at the point of selection has proven methodologically challenging and expensive (Author \& Author, 2017; Bieri \& Schuler, 2011; Rimm-Kaufman \& Hamre, 2010). In this article, we describe the development and administration of an online screening tool-a situational judgment test (SJT)—used to evaluate prospective teachers' non-cognitive attributes during the ITE selection process.

\section{Selection Methods for Teacher Education}

Selection methods for ITE are designed to evaluate program and professional suitability based on an assessment of personal characteristics (cognitive and non-cognitive attributes) and background factors, such as academic qualifications and relevant experiences. The urgency of the need to develop reliable and valid ITE selection processes varies across countries, ITE programs, and subject areas (Davies et al., 2016; Greenberg, Walsh, \& McKee, 2015; Ingvarson \& Rowley, 2017), with some programs facing a shortage of applicants and other programs needing to make difficult decisions about which applicants to select. However, even in settings where concerns about recruitment outweigh concerns about selection, using reliable, valid, and fair methods to assess applicants' cognitive and non-cognitive attributes can lead to a more robust understanding of the factors that influence teacher development.

During the ITE selection process, the cognitive attributes evaluated might include subject-area knowledge and general academic aptitude; the non-cognitive attributes 
assessed might include personality, beliefs, attitudes, motivation, and interpersonal skills. In Kunter et al.'s COACTIV model of professional competence (Kunter, Kleickmann, Klusmann, \& Richter, 2013), teachers' attributes play an important, but not exclusive, role in influencing professional competence and practices. Professional competence developed during an ITE program is influenced not only by personal characteristics, but also by the quality of learning opportunities (i.e., subject and pedagogical support), and diverse environmental factors (e.g., nature of in-school support during teaching placements). At the point of selection into ITE, it is candidates' personal characteristics that are under scrutiny. Assessment of cognitive attributes can be assessed in a relatively straightforward manner: academic transcripts and scores from tests of academic aptitude (e.g., Praxis) are widely available. Assessment of non-cognitive attributes is more challenging, with less agreement about what to assess and which methods to use in the hope of selecting candidates with the highest probability of success.

Selection methods vary in terms of cost, ease-of-use, candidate reactions, fairness, and potential to predict targeted outcomes, but recent research from organizational psychology and medical education suggests there are compelling reasons to choose some selection methods over others. A recent review of selection methods for medical education (Patterson et al., 2016) found that for measurement of cognitive attributes, academic records and performance on aptitude tests (e.g., MCAT and UKCAT) usefully predict medical school performance and success in professional practice. For assessment of non-cognitive attributes, assessment centers and situational judgment tests (SJTs) were adjudged to be more effective (higher predictive validity) and fairer (less prone to unconscious biases) than reference letters, personal statements, and traditional interviews. Assessment centers, which use a modular approach to selection, incorporating a series of tests, exercises, and structured interviews to assess non-cognitive attributes, are frequently used in business settings, and show high levels of predictive validity (Lievens \& Sackett, 2017). However, assessment centers are expensive to conduct, and are not feasible with large numbers of applicants. For large-scale selection, a screening process may be necessary to manage the numbers of candidates evaluated. In this article, we report the development and testing of a an SJT that can be used as one component of a screening process to assess applicants' non-cognitive attributes for selection into ITE.

\section{Situational Judgment Tests (SJTs)}

SJTs are a measurement method in which test-takers rate the appropriateness of responses to context-rich scenarios. A typical SJT item will consist of a brief, contextualized scenario that presents a challenging social situation, followed by the question What should (or would) you do? and a series of response options. SJTs are theorized to assess the testtaker's implicit trait policies (ITPs), or evaluation of the costs and benefits of particular courses of action. ITPs are based on underlying traits and beliefs gained from fundamental socialization processes (i.e., from family, schooling, peers, etc.). For example, in an SJT scenario targeting the personality trait of agreeableness, people will rely on their ITPs to make decisions about the most appropriate level of cooperation or competitiveness required for effective action in a given situation (Lievens \& Motowidlo, 2016). These internalized policies inform the internal decision-making about the best course of action in a challenging situation.

SJTs also assess procedural knowledge and situational judgment. Test-takers with specific knowledge and experience in a setting, e.g., ITE applicants with extensive experience working in schools, can draw on their knowledge about the most effective courses of action in a setting, but SJTs are more than assessments of previous experience; they also assess a candidate's situational judgment, independent of experience in a specific professional context (Melchers \& Kleinmann, 2016). Situational judgment refers to the ability to perceive and interpret an ambiguous social situation, with research showing that 
situational judgment is a valid predictor of a range of performance outcomes (Rockstuhl, Ang, Ng, Lievens, \& Van Dyne, 2015).

The popularity of SJTs among organizational psychologists and selection panels is based on their relatively high levels of predictive validity, their ease-of-use, cost effectiveness once developed, and generally favourable applicant reactions (e.g., Author et al., 2017; Lievens \& Motowidlo, 2017). In some ways, SJTs are similar to structured interview questions used in many ITE selection settings, but with the added advantages of gathering many more samples of candidate judgment and a more systematic and objective scoring system. In a face-to-face interview, candidates might be asked to respond to one or two contextualized scenarios, whereas in an SJT, responses to a high number of scenarios can be collected (i.e., 25 in the SJT in this study) in a relatively brief period of time. In addition, SJTs use a structured scoring system that is applied in the same way across candidates, with less possibility that the unconscious biases which influence face-to-face interview scoring (based on age, race, sexuality, class, gender, social background, and physical attractiveness) will affect the scoring (Cook, 2009).

Contextualized SJTs can be expensive and time-consuming to create, and in order to be relevant, reliable, and valid, their development requires the collaboration of test developers with subject matter experts (SMEs) who are familiar with the professional challenges in the field of interest (Patterson et al., 2015). Due to SJTs' multidimensional nature, the factor structure of SJTs can be ambiguous, with factor analysis typically revealing multiple uninterpretable factors (Lievens, Peeters, \& Schollaert, 2008). In spite of the psychometric challenges and expense of test development, SJTs are increasingly used to assess non-cognitive attributes for selecting candidates for training in a wide range of professional fields (Buyse \& Lievens, 2011; Taylor, Mehra, Elley, Patterson, \& Cousans, 2016). Longitudinal validity studies show that SJTs developed to test medical school applicants' non-cognitive attributes reliably predicted professional effectiveness several years after the selection process (Lievens \& Sackett, 2012). SJTs were rated as more effective for screening candidates for medical education than aptitude tests, personal statements, reference letters, and personality tests (Patterson et al., 2015). Research on SJTs for selection has been conducted in multiple professional fields, but use of the methodology for selection into teacher education has received only modest attention, and research on the efficacy of selection into ITE programs has been modest (Author \& Author, 2018).

\section{Current Study}

Our article has two objectives. The first objective, reported in Phase 1, is to describe the development of a construct-informed SJT used for ITE selection. The second objective, reported in Phases 2 and 3, is to report the results from the administration of the SJT to screen applicants for a day-long assessment center as part of the ITE selection process. The primary research questions are:

1. What are the psychometric properties of the teacher selection SJT (reliability, concurrent and predictive validity, factor structure, and statistical relationship with screening and assessment center activities)?

2. Does the SJT provide incremental predictive validity beyond screening methods currently used?

3. Do high scorers on the SJT fare better on assessment center activities than low scorers on the SJT?

4. Are there group differences (gender, SES) on the SJT scores?

Phase 1: Development of a Construct-Informed SJT for Screening ITE Applicants Participants in the development phase of the SJT were 19 SMEs (13 females, 6 males) who were involved in administering the ITE selection activities ('SME' was defined as (a) experience as a teacher, (b) experience as an interviewer on the selection process, or (c) experience with systematic observation of novice teachers). The SMEs worked with the research team to identify key attributes, develop the test specification, develop and review 
test items, and set the scoring key for the SJT. The development activities were conducted between 2015 and 2017.

Identifying target attributes. The process for identifying target attributes of the SJT followed an integrated inductive and deductive approach (e.g., Guenole, Chernyshenko, \& Weekly, 2017; Schubert et al., 2008; Weekley, Ployhart, \& Holtz, 2006). The majority of SJTs are developed using an inductive approach where key attributes are identified during the content development process (Campion, Ployhart, \& MacKenzie, Jr., 2014). In this approach, researchers work with SMEs to identify critical incidents related to the field of interest, and subsequently assign inductive categories to the content. In contrast, SJTs developed using a deductive approach identify target attributes before the content development process and develop content that represents the targeted attributes (e.g., Guenole et al., 2017). We used an integrated 'construct-informed' inductive and deductive approach, in which three noncognitive attributes emerged from a series of interviews with SMEs (i.e., 'bottom-up'), and three non-cognitive attributes were targeted a priori based on existing theories ('top-down'). The three inductive attributes_adaptability, organization, and empathy—were previously developed through a multi-step inductive process reported in Author et al., 2014, 2017. The three deductive attributes - conscientiousness, growth mindset, and emotion regulationwere chosen through a review of relevant literature and through a series of discussions with ITE program staff. Conscientiousness was chosen as a target attribute because it has been shown to be the Big Five personality domain most related to teacher effectiveness (Kim, DarNimrod, \& MacCann, 2018); growth mindset was chosen because of the increasing recognition that teachers' beliefs influence how students perceive their learning (e.g., Seaton, 2017), and emotion regulation was chosen because teacher emotions and emotion regulation are related to a range of important teaching-related outcomes (e.g., Chang, 2013; Sutton, 2004; Taxer \& Gross, 2018). The six non-cognitive attributes were used as a guide in the creation of scenarios of the SJT.

Test specification. The test specification-(a) purpose of the test, (b) test content, (c) item types, (d) response formats used, and (e) desired length of the test-was developed in a cooperation between the research team and key members of the ITE program. The (a) purpose of the test was to provide an initial online screening of applicants to an ITE program. The (b) test content was developed to evaluate applicant judgment related to six noncognitive attributes (i.e., conscientiousness, growth mindset, emotion regulation, adaptability, organization, and empathy). The (c) item type was determined to be scenarios of challenging classroom situations followed by response options. The (d) response format was a 4-point Likert rating scale indicating degree of appropriateness ('inappropriate' to 'appropriate'). As recommended by Whetzel and McDaniel (2016), we used 'should' instructions rather than 'would' instructions (What should you do in this situation?) in order to reduce candidate faking, since with this format all respondents have the same goal: to identify the best course of action in a particular context. The (e) desired length of the test was determined to be 30 minutes or less.

Item development. The 19 SMEs were interviewed by three members of the research team using a critical incident approach (e.g., Buyse \& Lievens, 2011). Participants in this phase were given the following written instructions (abridged) one week before individual meetings:

We are developing a tool that focuses on evidence-based attributes shown to be associated with successful teaching (definitions were provided for conscientiousness, emotion regulation, and mindset). During our face-to-face conversation, we will ask you to share two scenarios or incidents that are related to these attributes. These scenarios should relate to situations novice teachers might be expected to deal with, and the incident must relate to one (or more) of the six target attributes. We will also ask you to provide potential responses to the scenario and to rate the appropriateness of each response. 
The interviews were scheduled to last 45 minutes, and the researchers recorded scenarios and responses on an item development template. A total of 48 items were generated over two days of interviewing, with most items accompanied by five response options (range: 4-8 response options).

Review panel. The 48 items created in the item development phase were initially reviewed by the research team who edited the scenarios to eliminate errors, inappropriate items, redundant items, and items that did not clearly map onto the target attributes ${ }^{1}$. The SJT items were administered to a review panel consisting of subject matter experts SMEs from the ITE program who completed the test (and provided additional comments). The initial scoring key was developed using a mode consensus approach (Weng, Yang, Lievens, \& McDaniel, 2018), with item response options reduced to four. Items showing a high degree of consensus were retained, whereas items with a low degree of consensus were retained for further development. The final 25 -item SJT included items that were influenced by all six target attributes, but the distribution was not equally divided among the attributes: emotion regulation 10 items, conscientiousness 6 items, growth mindset 3 items, empathy 3 items, adaptability 2 items, and organization 1 item.

Format and scoring. The SJTs were designed to be delivered online as part of the initial screening activities completed by all applicants to the ITE program. The scoring key for the SJT was developed using a hybrid scoring approach where expert-based scoring was used to set the initial key, but scoring key adjustments were made empirically (Bergman, Donovan, Drasgow, Henning, \& Juraska, 2006), developed from a consensus among the review panel SME ratings. Item scores were calculated using a distance-measure approach, where a score is calculated based on distance from SME-determined correct score (with three points for a correct response, two points for a response one position away from correct, one point for a score two positions away from correct, and zero for a response three positions away from correct). Thus, the maximum total score was 300 ( 25 scenarios, 4 response options $\times 3$ maximum points for each response).

\section{Phase 2: Administration of a Prototype SJT to ITE Applicants}

The online screening process (Stage 1 selection) for this program runs on a nearcontinuous basis throughout the year, with on-site assessment centers (Stage 2 selection) scheduled at intermittent points. Data from the prototype SJT reported in Phase 2 were collected in 2017 and early 2018. The SJT was administered alongside the established ITEdeveloped screening process, but was not used for selection decisions.

Participants. The 3341 online applicants were $64.1 \%$ female, with a mean age of 26.49 years $(S D=13.10)$. Eligibility for free school meals $(F S M)$ was used as the first proxy for applicants' socio-economic background (e.g., llie, Sutherland, \& Vignoles, 2017): 23.9\% of applicants reported eligibility for FSM during their school years (in comparison, approximately $18 \%$ of UK primary school students were eligible for FSM in $2013 ; 13.7 \%$ in 2018; Department for Education, 2018). The other SES proxy was completion of higher education degree. In this sample, $40.8 \%$ of participants reported that their parents had completed a higher education degree.

Procedure. The online screening phase is designed to select candidates for the onsite full day assessment center. In this program, applicants for primary and secondary teacher training complete the same application process, and there is no differentiation in the process or activities used for selection.

Online screening process. Applicants completed three tasks for the online application: an eligibility check, three competency-based written questions, and the SJT. The

\footnotetext{
${ }^{1}$ A second version of the 25 -item SJT was developed and administered to a separate sample in parallel with the test reported in this article; however, technical problems in the scoring of the second version resulted in unusable data and results are not reported in this article.
} 
eligibility check ensured that applicants had a relevant previous degree and were eligible for teacher training in the UK (A-levels in relevant subjects, a Grade C or equivalent in GCSE Maths and English, and an undergraduate degree in a relevant teaching subject).

Participants completed the tasks at their convenience on the device of their choice (computer $87 \%$, tablet $1 \%$, and phone $8 \%$; with $4 \%$ not reported).

The three competency-based questions consisted of 250-word free responses to prompts on (a) understanding of education and motivation for teaching, (b) leadership potential, and (c) a third competency chosen by the candidate (not included in these analyses due to the varied nature of the topics chosen). The score for each question was calculated as the mean score from two raters using an 8-point scale for each question.

Decisions for invitation to the assessment center were based on the scores from the screening questions alongside a review of academic qualifications and other contextual recruitment information.

Situational judgment test. The Teacher Selection SJT consisted of 25 items delivered online during the Stage 1 online screening session. The test was prefaced with the following instructions: In this test, you are presented with scenarios that teachers encounter. Rate the appropriateness of each of the options in terms of what a (beginning teacher) should do (Inappropriate, Somewhat inappropriate, Somewhat appropriate, Appropriate), given the circumstances described in the scenario. There can be tied rankings, i.e., you can give multiple responses the same rating. Your rating on one option is independent from your ratings on the other options. For the test there are 25 questions, which should take 30 minutes to complete. Good luck!!

Applicants were not given a time limit for SJT completion, and applicants were not proctored during the test, i.e., they completed the test at the place and time, and on the device of their choosing. Figure 1 provides a sample SJT item (similar, but not identical to items administered as part of the screening process).

Assessment center. Applicants who scored above a certain threshold and who met other selection criteria (e.g., positive evaluation of related experiences, relevant teaching subject) were invited to attend the full-day, intensive assessment center. In Phase 1, 831 out of 3341 applicants $(24.9 \%)$ were invited to attend the assessment center. Activities at the assessment center included a competency-based 1-1 interview, a group activity centered around a case study, and a 7-minute sample teaching demonstration. Each of the three activities was scored out of 40 , with five competencies each scored out of 8 . Final decisions about acceptance to the program were based on assessment center scores plus a consideration of other relevant factors (e.g., teaching subject area).

Analysis. The analysis of the prototype SJT included reliability analysis, correlation coefficients with screening and assessment center activities, and analysis of individual items of the SJT.

\section{Phase 2 Results}

Brief summary results from analysis of the prototype version of the SJT are presented in this section, with a more detailed analysis of the revised SJT presented in Phase 3 results. The internal consistency (coefficient alpha) of the prototype SJT was .69, and bivariate correlations with the screening activities were $r=.28, p<.001$ with understanding and motivation score, and $r=.25, p<.001$ with the leadership score. Correlation of the prototype SJT with assessment center scores were $r=.07, p=.01$ with interview, $r=.10, p=.001$ with group case study, $r=.10, p=.001$ with the sample teaching scores, and $r=.13, p=.001$ with total assessment center score.

\section{Phase 3: Administration of Revised Version of SJT to ITE Applicants}

In Phase 3, we first reviewed and refined the SJT content based on item analysis, and then administered the revised version of the SJT to applicants who applied to the ITE program during a 3-month period in mid-2018. 
Test revision and item refinement. Scenarios and response options were reviewed and refined based on (a) item difficulty, (b) low item-total correlations, (c) negative correlations with outcomes, and (d) ambiguous response scoring. The review process identified five items that were deemed to benefit from revision. For example, items with toohigh or too-low item difficulty (i.e., did not discriminate among test-takers) were improved by reducing ambiguity of the response options.

Participants. Participants in Phase 3 were 587 applicants $(61.7 \%$ female; $23.0 \%$ eligible for FSM; $40.7 \%$ parents with higher education degrees) who completed an online SJT as part of the screening process. Of the 587 applicants, 97 (16.5\%) were invited to attend the on-site assessment center.

Procedure. The procedure for Phase 3 was identical to the procedure described in Phase 2.

Analysis. Analysis of the data comprised a descriptive analysis of means, range, and standard deviations for the key variables; assessment of online screening tasks for mean differences by gender and SES (defined as eligibility for free school meals); ANCOVA to examine differences on assessment center performance by SJT scoring group; analysis of bivariate associations between SJT scores and key variables; and hierarchical multiple regression showing the contribution of screening scores to prediction of assessment center activities.

\section{Phase 3 Results}

The results from the revised SJT were assessed for reliability, group differences, factor structure, and associations with screening and assessment center selection tools. An analysis of the five revised items showed improvements in item-total correlations and bivariate relationships with screening and assessment centre scores.

Internal consistency of the revised SJT was calculated using Cronbach's alpha, with a reliability coefficient of 0.78 . In Table 1 , we present the means, ranges, and standard deviations for the relationship between the SJT, screening scores (the two screening written questions, plus total screening score), and assessment center scores (for individual interview, group case study, sample teaching demonstration, and total assessment center score). Bivariate correlations with the screening activities were $r=.30, p<.001$ with understanding and motivation score, $r=.30, p<.001$ with the leadership score, and $r=.35$, $p<.001$ with total screening score. Correlation of the revised SJT with assessment center scores were $r=. .42, p<.001$ with interview, $r=.20, p=n s$ with group case study, $r=.48, p$ $<.001$ with the sample teaching scores, and $r=.46, p<.001$ with total assessment center score.

In order to test the factor structure of the SJT, we first conducted principal components analysis with oblimin rotation. As expected (see Lievens et al., 2008 on factor structure of construct-heterogeneous SJTs), the resulting factor structure was not easily interpretable in line with our target attributes or item content. We followed the advice of Fröhlich, Kahmann, and Kadmon (2017) to further investigate the factor structure using a minimum average partial test (MAP), more appropriate for construct-heterogeneous tests such as SJTs. The MAP test is conducted by partialing each factor out of the correlation matrix and calculating a partial correlation matrix. The number of factors to be retained is indicated where the average squared partial correlation reaches a minimum level (Velicer, Eaton, \& Fava, 2000). The results of the MAP test showed a single factor solution with an eigenvalue of 2.78 , suggesting a uni-dimensional structure.

We compared the scores from screening for differences according to SES (measured as eligibility for free school meals [FSM] and parental education level), gender, and type of device used to complete the screening activities. For the SJT, there were no significant differences for applicant SES measured by FSM, $F(1,390)=.15, p=.70 ; M_{H I G H}=242.74$, $S D_{H I G H}=14.26 ; M_{L O W}=242.19, S D_{L O W}=10.14$, or parental education level $F(1,455)=.037$, $p=.85$. For total screening score, there was a significant mean differences for SES as 
measured by FSM, $F(1,298)=5.53, p=.02, \eta_{\mathrm{p}}^{2}=.02 ; M_{\text {HIGH }}=14.57, S D_{H I G H}=3.36 ; M_{L O W}=$ 13.60, $\left.S D_{\text {LOW }}=3.35\right)$, but not for parental education level, $F(1,348)=.95, p=.33$.

Significant gender differences at screening were found for SJT scores $F(1,480)=$ 8.67, $p=.003, \eta_{\mathrm{p}}^{2}=.02$ (considered 'small'; Cohen, 1988); $M_{F}=242.6, S D_{F}=10.01 ; M_{M}=$ 238.85, $S D_{M}=18.08$, but not for total screening score $F(1,361)=1.56, p=.21, M_{F}=14.24$, $S D_{F}=3.48 ; M_{M}=13.77, S D_{M}=3.58$. Applicants who used a computer to complete the screening activities scored significantly higher, $F(3,361)=4.91, p=.002)$ on the written responses to competency-based questions $(M=14.34, S D=3.45)$ than applicants who used a phone $(M=12.0, S D=3.40$, but there were no significant differences on SJT scores by type of device used $(F(3,480)=1.64, p=.18)$.

We divided participants into three equal groups (high, medium, and low scorers) according to SJT scores. A one-way ANCOVA was conducted to determine the effect of SJT score on assessment center scores $\left(M_{\mathrm{HIGH}}=87.82, M_{\mathrm{MED}}=75.47, M_{\mathrm{LOW}}=69.59\right)$ controlling for gender and SES (using FSM as proxy). There was a significant effect of SJT score on assessment center score $F(2,62)=12.37, p<.001, \eta_{p}^{2}=.30$, considered a large effect size (Cohen, 1988).

In Tables 2-5, we report the bivariate correlations between SJT scores, screening scores, and assessment center scores. In Table 2, we see that SJTs were significantly associated with both screening activities (and total screening score), and with 4 out of 5 assessment center scores, including offer decision $(r=.34, p<.01)$. SJTs were not significantly associated with the assessment center group case study activity $(r=.20, p=$ ns). Tables 3-5 report the association of SJT scores with individual categories within each of the assessment center activities. In Table 3, SJT scores were significantly correlated with each of the five categories of the interview ( $r$ ranging from $.26-.39$ ), and more strongly correlated than the total screening score for each of the categories. In Table 4, we see that SJTs were only weakly associated with scores from the group case study activity (rs ranging from .06 - .25). In Table 5, we see that SJT scores were significantly associated with all categories of the sample teaching scores ( $r$ s ranging from .37-.45), and were more strongly associated with teaching scores than was the total screening score ( $r$ ranging from .04 $.23)$.

Table 6 reports the results of hierarchical multiple regression analyses testing how SJTs incrementally predicted assessment center activities after accounting for the screening scores. At Step 1, total screening score made a significant contribution to the prediction of all three assessment center activities, with $\beta$-weights ranging from .22 to .26 . The addition of SJT scores in Step 2 added incremental predictive validity to the prediction of Interview scores $\left(\Delta R^{2}=.15, p<.01\right)$ and for the sample teaching $\left(\Delta R^{2}=.20, p<.01\right)$, but not for the group case study $\left(\Delta R^{2}=.02, p=n s\right)$. All three regression equations were significant: interview score, $R^{2}=.20, F(2,94)=11.80, p<.01$; group case study, $R^{2}=.09, F(2,94)=$ $4.47, p=.01$; sample teaching, $R^{2}=.24, F(2,94)=14.63, p<.01$.

The $\beta$-weight for the SJT was stronger than the $\beta$-weight for the screening score for two out of the three assessment center activities: interview score $\left(\beta_{\mathrm{SJT}}=.40, p<.01\right.$; $\left.\beta_{\text {SCREENING }}=.12, p=n s\right)$, and sample teaching ( $\beta_{\text {SJT }}=.46, p<.01 ; \beta_{\text {SCREENING }}=.07, p=n s$ ). However, the $\beta$-weight for screening score was a better predictor of the group case study score than was the SJT ( $\left.\beta_{\text {SJT }}=.15, p=n s ; \beta_{\text {SCREENING }}=.21, p<.05\right)$.

\section{General Discussion}

We reported the development and validation of an online, construct-informed SJT to screen candidates who applied for a large and selective teacher education program in the UK. Results from the study suggest that the SJT was internally consistent, showed concurrent validity with other screening methods, and predicted candidates' performance at the intensive assessment center used for final selection. Gender differences $(F>M)$ were more pronounced in the SJT than in other screening methods, but SES differences were not significant in the SJT. The SJT factor structure did not align with the constructs used to 
develop the test, but instead represented a single factor assessing situational judgment. The SJT represents a new selection method in education, with administration and scoring that is cost-effective, efficient, and could be used as part of a suite of selection tools to screen candidates as part of a high-volume selection process, or to add novel information about candidates' situational judgment in smaller-scale selection processes.

Four research questions were posed in the study. In response to the first question, the psychometric properties of the revised SJT were strong, with acceptable internal consistency, and significant positive associations with concurrent screening and future assessment centre activities. We used a construct-informed approach to build SJT content, but the factor structure emerging from the principal components analysis was largely uninterpretable, and did not map cleanly on to the targeted non-cognitive attributes used to develop item content. This pattern of results is not uncommon in SJT research because each 'real-life' scenario, even when built to target a particular construct, reflects multiple constructs (Campion et al., 2014). Our MAP analysis gives us some confidence that the SJT measures a general construct of situational judgment, but the results raise issues about how the targeted constructs are represented in the scenarios developed for the test.

The answer to the second question-does the SJT provide incremental predictive beyond current screening methods-was answered affirmatively, with evidence from hierarchical multiple regression analysis showing that scores on the SJT predict applicant performance at the assessment center. The links between SJT and assessment center interview and sample teaching demonstration were significant and positive; however, the SJT was less clearly associated with applicant scores on the group case study. It is likely that the individualized nature of the SJT (What should you do?) is less useful in predicting the group dynamics assessed in the group case study activity. The third question, pertaining to highand low-scorers on the SJT, is pertinent to decision-making based on test scores, with results showing that applicants who fared poorly on the SJT also fared poorly on the multiple activities that took place during the assessment centre.

The fourth research question pertained to group differences on SJT scores. Although the scores from the SJT did not differ according to SES background, there were significant differences on the SJT favouring females, and these differences were not found in the other screening methods. The direction of the gender difference has been seen in other SJT research (e.g., Whetzel, McDaniel, \& Nguyen, 2008), but is potentially troubling in a profession where recruiting and retaining males presents challenges in many education systems (e.g., Pollitt \& Oldfield, 2017). Further investigation into the reasons behind female applicants' better performance on SJTs is worth further scrutiny. Screening applicants for ITE relies on imperfect data, but adding scores from multiple sources, including SJTs, increases the likelihood of making better-informed and evidence-supported selection decisions.

The overall aim of selection procedures is to make decisions about the probability of applicants' future success, but selection methods range in cost (including time costs) and how effective they are in predicting success. Recent work by (Author et al., 2017) showed that the cost and predictive utility of teacher selection methods were statistically unrelated, with overall prediction of objectively measured (i.e., not self-reported) teacher effectiveness generally low. Assessing applicants' non-cognitive attributes in a systematic, cost-effective, objective, and efficient way during a selection process presents real challenges for ITE programs. Some selection methods that are used for screening for ITE-reference letters and personal statements, in particular-show little grounds for use in decision-making, and furthermore, may be prone to reviewers' conscious or unconscious biases (e.g., Mason \& Schroeder, 2014; Patterson et al., 2016). Using a modular approach to selection, where a suite of evidence-supported selection methods are incorporated into the selection process improves the quality-the reliability, validity, and fairness-of the decisions made (Lievens \& Sackett, 2017). 
One important question about selection is whether assessing non-cognitive attributes that may change over time are worth including in a selection process. There is evidence that the relationship between the attributes measured at selection and targeted outcomes may evolve. Blair and colleagues (Blair, Hoffman, \& Ladd, 2016) showed in a business setting that SJTs and general mental ability both significantly predicted work success one year after initial assessment, but performance in assessment centers did not. However, six years after the initial assessment, the contribution of general mental ability dissipated, SJTs continued to be related to work success (but to a lesser extent), and scores from assessment centers increased in their association with success. Similar results were found in selection into medicine and dentistry. Buyse \& Lievens (2011) found that the predictive validity of cognitive ability measured at selection dropped through the five years of dental training, whereas the predictive validity of SJTs designed to assess interpersonal skills increased from negligible in Year 1 to positive and significant in Year 5. Similarly, Lievens and Sackett (2012) showed that an interpersonal SJT administered at selection into medical education grew in importance up to nine years into professional practice. Non-cognitive attributes such as interpersonal skills, personality, and motivation may be nurtured during professional training, but the starting point-the core attributes measured at selection-appear to play an important role in future professional competence. For ITE programs, ignoring the evaluation of candidates' non-cognitive attributes, or using methods that lack an evidence base may be a mistake.

\section{Limitations}

The study does not address the longer-term predictive utility of SJT, and further work is needed to connect selection methods and longer-term teaching outcomes. In fact, very little is known about how methods used for teacher selection are related to important teaching outcomes (Goldhaber, Grout, \& Huntington-Klein, 2014). Our research shows a connection between a selection method used at screening, and candidates' performance at an assessment center, including on a teaching demonstration, but it is possible that actual teaching effectiveness is unrelated to the decisions made at selection. Even though we know that SJTs used for selection in professional fields tend to be positively related to professional outcomes (e.g., Lievens \& Sackett, 2012), what is less clear is which underlying constructs are contributing to the prediction of these outcomes. Our factor analytic results showed that the data did not separate cleanly into the six non-cognitive attributes on which the SJT was built. A next step in development of SJTs for teacher selection might be to build tools that focus on single, well-defined constructs such as integrity, emotional intelligence, and conscientiousness (see Libbrecht \& Lievens, 2012 for a review). However, the hallmark of SJTs is their real-world relevance, and one advantage to using contextualized situations (i.e., a challenging classroom scenario) to evaluate judgment is that how people enact their personality, beliefs, and motivations is dependent on contextual factors (e.g., Chen, Fan, Zheng, \& Hack, 2016).

\section{Conclusion}

In summary, the results from this research suggest that a teaching-focused SJT could be a useful tool to assess certain aspects of candidates' non-cognitive attributes, and especially situational judgment, when they apply to ITE programs. Although not frequently included in selection methods in education, the wealth of evidence demonstrating the reliability, validity, and robustness of SJTs used for selection in health- and business-related fields suggests that this methodology should receive serious consideration when ITE selection methods are developed. A teaching-focused SJT may be especially useful in large-scale ITE selection procedures, where the cost-efficiency and ease-of-use are important considerations, but smaller-scale settings may also benefit from the contribution of information derived from a reliable, valid, and un-biased assessment of candidates' judgment in teaching situations. 


\section{References}

Arthur Jr., W., Glaze, R. M., Jarrett, S. M., White, C. D., Schurig, I., \& Taylor, J. E. (2014). Comparative evaluation of three situational judgment test response formats in terms of construct-related validity, subgroup differences, and susceptibility to response distortion. Journal of Applied Psychology, 99, 535-545.

Atteberry, A., Loeb, S., \& Wyckoff, J. (2015). Do first impressions matter? Predicting early career teacher effectiveness. AERA Open, 1, 1-23.

Author et al. (2017).

Author \& Author (2018).

Bastian, K. C., McCord, D. M., Marks, J. T., \& Carpenter, D. (2017). A temperament for teaching? Associations between personality traits and beginning teacher performance and retention. AERA Open, 3, 1-17.

Bergman, M. E., Donovan, M. A., Drasgow, F., Henning, J. B., \& Juraska, S. E. (2006). Scoriing situational judgment tests: Once you get the data, your troubles begin. International Journal of Selection and Assessment, 14, 223-235.

Bieri, C., \& Schuler, P. (2011). Cross-curricular competencies of student teachers: a selection model based on assessment centre admission tests and study success after the first year of teacher training. Assessment \& Evaluation in Higher Education, 36, 399415.

Blair, C. A., Hoffman, B. J., \& Ladd, R. T. (2016). Assessment centers vs situational judgment tests: longitudinal predictors of success. Leadership \& Organization Development Journal, 37, 899-911.

Bowles, T., Hattie, J., Dinham, S., Scull, J., \& Clinton, J. (2014). Proposing a comprehensive model for identifying teaching candidates. The Australian Educational Researcher, 41, 365-380.

Buyse, T., \& Lievens, F. (2011). Situational judgment tests as a new tool for dental student selection. Journal of Dental Education, 75, 743-749.

Campion, M. C., Ployhart, R. E., \& MacKenzie, W. I. (2014). The state of research on situational judgment tests: A content analysis and directions for future research. Human Performance, 27, 283-310.

Chang, M. L. (2013). Toward a theoretical model to understand teacher emotions and teacher burnout in the context of student misbehavior: Appraisal, regulation and coping. Motivation and Emotion, 37, 799-817.

Chen, L., Fan, J., Zheng, L., \& Hack, E. (2016). Clearly defined constructs and specific situations are the currency of SJTs. Industrial and Organizational Psychology, 9, 34-38.

Cohen, J. (1988) Statistical power analysis for the behavioral sciences (2nd ed.) Hillsdale, NJ: Erlbaum.

Cook, M. (2009). Personnel selection: Adding value through people (5th edition). Chichester, UK: Wiley-Blackwell. 
Davies, P., Connolly, M., Nelson, J., Hulme, M., Kirkman, J., \& Greenway, C. (2016). 'Letting the right one in': Provider contexts for recruitment to initial teacher education in the United Kingdom. Teaching and Teacher Education, 60, 291-302.

Department for Education (2018). Schools, pupils and their characteristics: January 2018.

Retrieved from

https://assets.publishing.service.gov.uk/government/uploads/system/uploads/attachment data/file/719226/Schools Pupils and their Characteristics 2018 Main Text.pdf

Fröhlich, M., Kahmann, J., \& Kadmon, M. (2017). Development and psychometric examination of a German video-based situational judgment test for social competencies in medical school applicants. International Journal of Selection and Assessment, 25, 94110.

Goldhaber, D., Grout, C., \& Huntington-Klein, N. (2014). Screen twice, cut once: Assessing the predictive validity of teacher selection tools. CEDR Working Paper No. 2014-9: University of Washington, Seattle, WA.

Greenberg, J., McKee, A., \& Walsh, K. (2015). Teacher prep review: A review of the nation's teacher preparation programs. National Council on Teacher Quality. Retrieved from http://www.nctq.org/dmsView/Teacher_Prep_Review_2014_Report.

Guenole, N., Chernyshenko, O. S., \& Weekly, J. (2017). On designing construct driven situational judgment tests: Somer preliminary recommendations. International Journal of Testing, 17, 234-252.

Ilie, S., Sutherland, A., \& Vignoles, A. (2017). Revisiting free school meal eligibility as a proxy for pupil socio-economic deprivation. British Educational Research Journal, 43, 253-274.

Ingvarson, L., \& Rowley, G. (2017). Quality assurance in teacher education and outcomes: A study of 17 countries. Educational Researcher, 46, 177-193.

Kim, L. E., Dar-Nimrod, I., \& MacCann, C. (2018). Teacher personality and teacher effectiveness in secondary school: Personality predicts teacher support and student selfefficacy but not academic achievement. Journal of Educational Psychology, 110, 309323.

Klassen, R. M., \& Tze, V. (2014). Teachers' self-efficacy, personality, and teaching effectiveness: A meta-analysis. Educational Research Review, 12, 59-76.

Kunter, M., Kleickmann, T., Klusmann, U., \& Richter, D. (2013). The development of teachers' professional competence. In M. Kunter, J. Baumert, W. Blum, U. Klusmann, S. Krauss, \& M. Neubrand (Eds.), Cognitive activation in the mathematics classroom and professional competence of teachers (pp. 63-77). New York, NY: Springer.

Libbrecht, N., \& Lievens, F, (2012). Validity evidence for the situational judgment test paradigm in emotional intelligence measurement. International Journal of Psychology, 47, 438-447.

Lievens, F. (2017). Construct-driven SJTs: Toward an agenda for future research. International Journal of Testing, 269-276. 
Lievens, F., \& Motowidlo, S. J. (2016). Situational judgment tests: From measures of situational judgment to measures of general domain knowledge. Industrial and Organizational Psychology, 9 3-22.

Lievens, F., Peeters, H., \& Schollaert, E. (2008). Situational judgment tests: a review of recent research. Personnel Review, 37, 426-441.

Lievens, F., \& Sackett, P. R. (2012). The validity of interpersonal skills assessment via situational judgment tests for predicting academic success and job performance. The Journal of Applied Psychology, 97, 460-468.

Lievens, F., \& Sackett, P. R. (2017). The effects of predictor method factors on selection outcomes: A modular approach to personnel selection procedures. Journal of Applied Psychology, 102, 43-66.

Mason, R. W., \& Schroeder, M. P. (2014). The predictive validity of teacher candidate letters of reference. Journal of Education and Learning, 3, 67-75.

Matoskova, J., \& Kovarik, M. (2016). Development of a situational judgment test as a predictor of college student performance. Journal of Psychoeducational Assessment, 35, 768-784.

McDaniel, M. A., List, S. K., \& Kepes, S. (2016). The "hot mess" of situational judgment test construct validity and other issues. Industrial and Organizational Psychology, 9, 47-51.

Melchers, K. G., \& Kleinmann, M. (2016). Why situational judgment is a missing component in the theory of SJTs. Industrial and Organizational Psychology, 9, 29-34.

OECD (2005). Teachers matter: Attracting, developing and retaining effective teachers.

Paris, France: OECD Publishing. Retrieved from

http://www.oecd.org/education/school/attractingdevelopingandretainingeffectiveteachersfinalreportteachersmatter.htm

Patterson, F., Knight, A., Dowell, J., Nicholson, S., Cousans, F., \& Cleland, J. (2016). How effective are selection methods in medical education? A systematic review. Medical Education, 50, 36-60.

Pollitt, K., \& Oldfield, J. (2017). Overcoming the odds: Exploring barriers and motivations for male trainee primary teachers. Teaching and Teacher Education, 62, 30-36.

Rimm-Kaufman, S. E., \& Hamre, B. K. (2010). The role of psychological and developmental science in efforts to improve teacher quality. Teachers College Record, 112, 2988-3023.

Rockoff, J. E., Jacob, B. A., Kane, T. J., \& Staiger, D. O. (2011). Can you recognize an effective teacher when you recruit one? Education Finance and Policy, 6, 43-74.

Rockstuhl, T., Ang, S., Ng, K.-Y., Lievens, F., \& Van Dyne, L. (2015). Putting judging situations into situational judgment tests: Evidence from intercultural multimedia SJTs. Journal of Applied Psychology, 100, 464-480.

Schubert, S., Ortwein, H., Dumitsch, A., Schwantes, U., Wilhelm, O., \& Kiessling, C. (2008). A situational judgement test of professional behaviour: development and validation. Medical Teacher, 30, 528-533. 
Seaton, F. S. (2017). Empowering teachers to implement a growth mindset. Educational Psychology in Practice, 34, 41-57.

Sutton, R. E. (2004). Emotional regulation goals and strategies of teachers. Social Psychology of Education, 7, 379-398.

Taxer, J. L., \& Gross. J. J. (2018). Emotion regulation in teachers: The "why" and "how". Teaching and Teacher Education, 74, 180-189.

Taylor, N., Mehra, S., Elley, K., Patterson, F., \& Cousans, F. (2016). The value of situational judgement tests for assessing non-academic attributes in dental selection. British Dental Journal, 220, 565-566.

Velicer, W. F., Eaton, C. A., \& Fava, J. L. (2000). Construct explication through factor or component analysis: A review and evaluation of alternative procedures for determining the number of factors or components. In R. Goffin, \& E. Helmes (Eds.), Problems and solutions in human assessment: Honoring Douglas N. Jackson at seventy (pp. 41-71). Boston, MA: Kluwer.

Watt, H. M. G., Richardson, P. W., \& Wilkins, K. (2014). Profiles of professional engagement and career development aspirations among USA preservice teachers. International Journal of Educational Research, 65, 23-40.

Weekley, J. A., Ployhart, R. E., \& Holtz, B. C. (2006). On the development of situational judgment tests: Issues in item development, scaling, and scoring. In J. A. Weekley \& R. E. Ployhart (Eds.), Situational judgment tests: Theory, measurement and application (pp. 157-182. Mahwah, NJ: Lawrence Erlbaum Associates.

Weng, Q., Yang, H., Lievens, F., \& McDaniel, M. A. (2018). Optimizing the validity of situational judgment tests: The importance of scoring methods. Journal of Vocational Behavior, 104, 199-209.

Whetzel, D. L., \& McDaniel, M. A. (2016). Are situational judgment tests better assessments of personality than traditional personality tests in high-stakes testing? In U. Kumar (Ed.), The Wiley handbook of personality assessment (pp. 205-214). Chichester, UK: Wiley \& Sons.

Whetzel, D. L., McDaniel, M. A., \& Nguyen, N. T. (2008). Subgroup differences in situational judgment test performance: A meta-analysis. Human Performance, 3,

Xu, Z., Özek, U., \& Hansen, M. (2015). Teacher performance trajectories in high-and lowerpoverty schools. Educational Evaluation and Policy Analysis. Retrieved from http://epa.sagepub.com/content/37/4/458.short 
Table 1

Descriptive Statistics for SJT, Screening Scores, and Assessment Centre Scores

\begin{tabular}{lcll}
\hline & $N$ & $M$ (Range) & $S D$ \\
\hline SJT & 587 & $240.75(117.0-264.0)$ & 13.69 \\
Understanding and motivation (screening) & 449 & $4.66(1-8)$ & 1.26 \\
Leadership (screening) & 449 & $4.76(1-8)$ & 1.32 \\
Total screening & 449 & $14.04(3-22)$ & 3.40 \\
AC interview & 97 & $28.07(13-40)$ & 5.33 \\
AC case study & 97 & $25.25(1-39)$ & 6.36 \\
AC sample teaching & 97 & $26.15(0-41)$ & 7.48 \\
Total AC & 97 & $79.47(14-104)$ & 15.54 \\
\hline
\end{tabular}


Table 2

Correlations Between SJT, Screening Scores, Assessment Centre Scores, and Offer

\begin{tabular}{|c|c|c|c|c|c|c|c|c|c|}
\hline & 1 & 2 & 3 & 4 & 5 & 6 & 7 & 8 & 9 \\
\hline 1. SJT & - & $.30^{* *}$ & $.30^{* *}$ & $.35^{* *}$ & $.42^{* *}$ & .20 & $.48^{* *}$ & $.46^{* *}$ & $.34^{* *}$ \\
\hline 2. Understanding and motivation (screening) & & - & $.58^{* *}$ & $.82^{* *}$ & .14 & $.24^{*}$ & .20 & $.24^{*}$ & $.25^{*}$ \\
\hline 3. Leadership (screening) & & & - & $.88^{* *}$ & $.29^{*}$ & .14 & .14 & $.22^{*}$ & $.26^{*}$ \\
\hline 4. Total screening & & & & - & $.24^{*}$ & $.26^{*}$ & $.22^{*}$ & $.29^{* *}$ & $.28^{* *}$ \\
\hline 5. AC Interview & & & & & - & $.46^{\star *}$ & $.40^{* *}$ & $.73^{* *}$ & $.53^{* *}$ \\
\hline 6. AC case study & & & & & & - & $.56^{\star *}$ & $.84^{* \star}$ & $.62^{* *}$ \\
\hline 7. AC sample teaching & & & & & & & - & $.85^{* *}$ & $.67^{* *}$ \\
\hline 8. Total AC & & & & & & & & - & $.76^{* *}$ \\
\hline 9. Offer & & & & & & & & & - \\
\hline
\end{tabular}

${ }^{\star} p<.01,{ }^{* *} p<.001$ 
Table 3

Correlations Between SJT, Screening Score, and Interview Scores

\begin{tabular}{|c|c|c|c|c|c|c|c|}
\hline & 1 & 2 & 3 & 4 & 5 & 6 & 7 \\
\hline 1. SJT & - & $.35^{* \star}$ & $.26^{* *}$ & $.35^{* *}$ & $.39^{* *}$ & $.33^{* *}$ & $.30^{* *}$ \\
\hline 2. Total screening & & - & .13 & $.28^{* *}$ & .10 & $.22^{*}$ & .19 \\
\hline 3. Interview (Understanding and motivation) & & & - & $.50^{* *}$ & $.48^{* *}$ & $.35^{\star *}$ & $.50^{* *}$ \\
\hline 4. Interview (Leadership) & & & & - & $.64^{* *}$ & $.43^{* *}$ & $.58^{* *}$ \\
\hline 5. Interview (Planning and organisation) & & & & & - & $.46^{\star *}$ & $.62^{* *}$ \\
\hline 6. Interview (Problem solving) & & & & & & - & $.35^{\star *}$ \\
\hline 7. Interview (Resilience) & & & & & & & - \\
\hline
\end{tabular}

${ }^{*} p<.01,{ }^{* *} p<.001$ 
Table 4

Correlations Between SJT, Screening Score, and Case Study Scores

\begin{tabular}{|c|c|c|c|c|c|c|c|}
\hline & 1 & 2 & 3 & 4 & 5 & 6 & 7 \\
\hline 1. SJT & - & $.35^{\star *}$ & .19 & $.20^{*}$ & .06 & .16 & $.25^{\star}$ \\
\hline 2. Total screening & & - & $.24^{*}$ & $.24^{*}$ & .14 & $.25^{\star}$ & $.25^{*}$ \\
\hline 3. Case study (Empathy) & & & - & $.81^{* *}$ & $.65^{* *}$ & $.60^{* *}$ & $.50^{* *}$ \\
\hline 4. Case study (Interaction) & & & & - & $.69^{* *}$ & $.66^{* *}$ & $.58^{* *}$ \\
\hline 5. Case study (Leadership) & & & & & - & $.71^{* *}$ & $.47^{* *}$ \\
\hline 6. Case study (Problem solving) & & & & & & 一 & $.51^{* *}$ \\
\hline 7. Case study (Self-evaluation) & & & & & & & - \\
\hline
\end{tabular}

${ }^{\star} p<.01,{ }^{* *} p<.001$ 
Table 5

Correlations Between SJT, Screening Score, and Sample Teaching Scores

\begin{tabular}{|c|c|c|c|c|c|c|c|}
\hline & 1 & 2 & 3 & 4 & 5 & 6 & 7 \\
\hline 1. SJT & - & $.35^{* *}$ & $.45^{* *}$ & $.40^{* *}$ & $.42^{* *}$ & $.37^{* *}$ & $.43^{* *}$ \\
\hline 2. Total screening & & - & $.23^{*}$ & $.21^{*}$ & $.22^{*}$ & $.21^{*}$ & .04 \\
\hline 3. Sample teaching (Empathy) & & & - & $.74^{* *}$ & $.61^{* *}$ & $.77^{* *}$ & $.60^{* *}$ \\
\hline 4. Sample teaching (Interaction) & & & & - & $.78^{* *}$ & $.84^{* *}$ & $.61^{* *}$ \\
\hline 5. Sample teaching (Planning and organisation) & & & & & - & $.68^{* *}$ & $.57^{* *}$ \\
\hline 6. Sample teaching (Resilience) & & & & & & - & $.61^{* *}$ \\
\hline 7. Sample teaching (Self-evaluation) & & & & & & & - \\
\hline
\end{tabular}

${ }^{\star} p<.01,{ }^{* *} p<.001$ 
Table 6

Hierarchical Multiple Regression Analyses Predicting Assessment Center Performance in Interview, Case Study, and Sample Teaching from Screening Scores and SJTs

\begin{tabular}{|c|c|c|c|c|c|c|}
\hline \multirow[b]{3}{*}{ Predictor } & \multicolumn{6}{|c|}{ Assessment Centre Activities } \\
\hline & \multicolumn{2}{|c|}{ Interview } & \multicolumn{2}{|c|}{ Case study } & \multicolumn{2}{|c|}{ Sample teaching } \\
\hline & $\Delta R^{2}$ & $\beta$ & $\Delta R^{2}$ & $\beta$ & $\Delta R^{2}$ & $\beta$ \\
\hline \multicolumn{7}{|l|}{ Step 1} \\
\hline Total screening & $.06^{*}$ & $.24^{*}$ & $.07^{*}$ & $.26^{*}$ & $.05^{*}$ & $.22^{*}$ \\
\hline \multicolumn{7}{|l|}{ Step 2} \\
\hline Total screening & & .12 & & $.21^{*}$ & & .07 \\
\hline SJT & $.15^{\star \star}$ & $.40^{* *}$ & .02 & .15 & $.20^{* *}$ & $.46^{* *}$ \\
\hline Total $R^{2}$ & $.20^{* *}$ & & .09 & & $.24^{* *}$ & \\
\hline$n$ & 97 & & 97 & & 97 & \\
\hline
\end{tabular}

${ }^{\star} p<.01,{ }^{* *} p<.001$ 
You are teaching a Year 9 science class and the students are listening as you explain something on the whiteboard. At one point, you forget what you want to say next. As you pause, a girl in the front row laughs and says, "You're useless!" but only loud enough so you and maybe some pupils next to her can hear.

Rate the appropriateness of each of the options in terms of what you should do as a first-year teacher:

Response options:

- Quietly and firmly ask the student to leave the class in order to establish your authority in front of the other students (Inappropriate)

- Quietly tell her that the comment was not acceptable and explain what consequence she will face (somewhat appropriate)

- Ignore her, gather your thoughts and carry on (somewhat appropriate)

- Turn and explain to the class what just happened, outline why it was inappropriate and what consequence she will face (somewhat inappropriate)

Figure 1. Example item from situational judgment test 